\title{
De Novo Synthesis of Benzannelated Heterocycles
}

\author{
Johannes Feierfeil and Thomas Magauer ${ }^{*[a]}$ \\ Dedicated to Professor Dr. Herbert Mayr on the occasion of his 70th birthday
}

\begin{abstract}
Benzannelated heterocycles such as indoles and indazoles are prominent structural motifs found in natural products, pharmaceuticals and agrochemicals. For their synthesis, chemists traditionally either functionalize commercially available heterocycles or resort to transformations that make use of benzene derived building blocks. Here, we report a powerful cascade reaction that enables the de novo construction of variously substituted indoles, indazoles, benzofurans and benzothiophenes from readily available bicyclo[3.1.0]hexan-2-ones. The transformation can be conducted under mild, non-anhydrous conditions. For the synthesis of indoles, mechanistic studies revealed that the electrocyclic ring-opening of the bicyclic ring-system and aromatization precedes the 3,3sigmatropic rearrangement.
\end{abstract}

\section{Introduction}

Five-membered benzannelated heterocycles are ubiquitous in biologically active molecules and are precious building blocks for the chemical industry (Figure 1). ${ }^{[1]}$ Conventional methods for the synthesis of substituted indoles and indazoles typically make use of either pre-functionalized heterocycles or rely on benzene-derived building blocks. Protocols that enable the de novo synthesis of these heterocycles are rare and often are associated with unsatisfactory functional group tolerance, efficiency, or practicability (e.g. use of stannanes, high temperatures, expensive transition metals). ${ }^{[2]}$ Examples (Figure 2a) include the Kanematsu indole synthesis, which involves a multistep sequence to access the allene precursor, requires high temperatures, and only tolerates alkyl and phenyl groups ${ }^{[3]}$ and the Pagenkopf indole synthesis, in which the indole core is constructed via a nitrile/donor-acceptor cyclopropane [3+2] cyclization. ${ }^{[4]}$ The Funk group also reported a $6 \pi-$ electrocyclization/condensation approach to construct the indole structural motif of the natural product welwistatin. ${ }^{[5}$

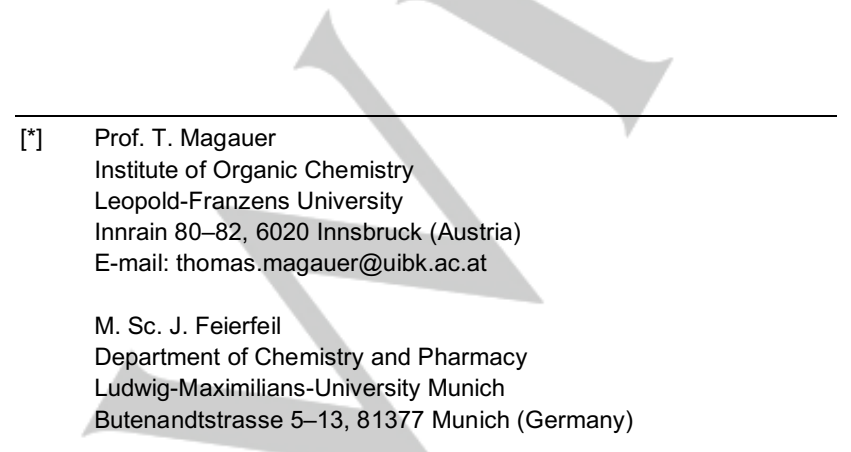

Supporting information for this article is given via a link at the end of the document.

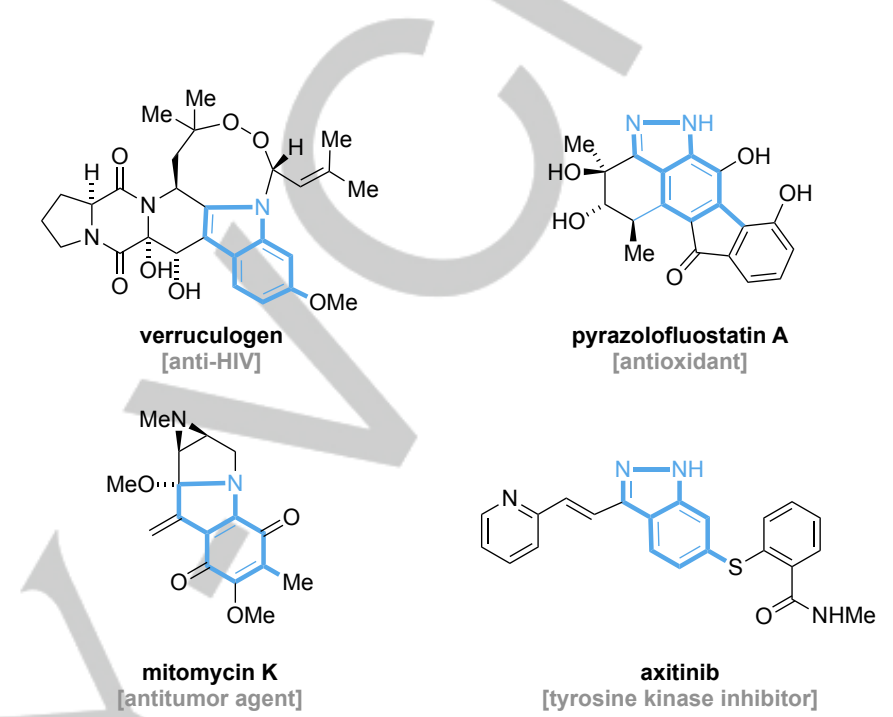

Figure 1. Selected examples of biologically active molecules featuring an C6functionalized indole or indazole structural motif.

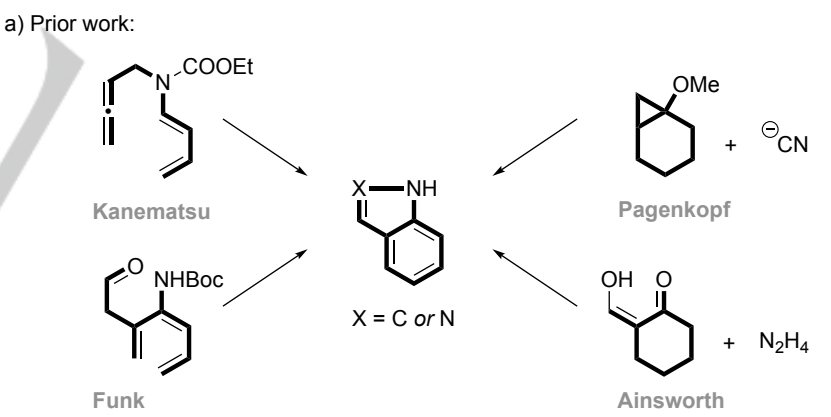

b) This work:

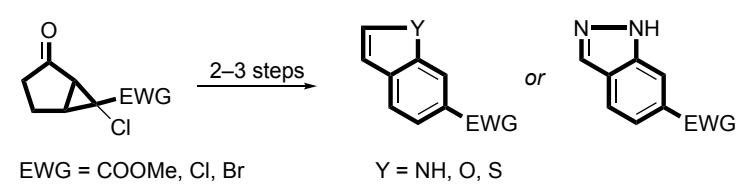

Figure 2. a) Selected methods for the synthesis of indoles and indazoles from non-aromatic precursors. b) De novo synthesis of benzannelated heterocycles from bicyclo[3.1.0]hexan-2-ones.

Another rare example for the synthesis of an indazole from a non-aromatic precursor was described by Ainsworth ${ }^{[6]}$ and involves the condensation of hydrazine with a $\beta$-hydroxyenone. A drawback of these strategies is the need for toxic oxidants such as 2,3-dichloro-5,6-dicyano-p-benzoquinone or the use of palladium in the final aromatization step. ${ }^{[7]}$ 
In recent work we demonstrated that bicyclic ring-systems can be efficiently converted to polyfunctionalized 3hydroxybenzoates ${ }^{[8]}$ and orthogonally functionalized, chlorinated naphthoates. ${ }^{\left[{ }^{[9]}\right.}$ Initial efforts to incorporate heteroatoms in the ring-opening precursor were largely unsuccessful and only trace amounts of the desired heterocycles could be obtained. Here, we report a solution to this problem and show its general applicability to the efficient syntheses of indoles, indazoles, benzofurans and benzothiophenes.

\section{Results and Discussion}

We began our investigations with O-vinyl oxime 1 , which was prepared in two steps from readily available methyl bicyclo[3.1.0]hexan-2-ones (see Supplementary Information for details). ${ }^{[10]}$ To identify the optimal conditions for the synthesis of indole 2, O-vinyl oxime 1 was subjected to an extensive screen of reaction conditions (Table 1). We found that conducting the reaction in sulfolane $(0.2-0.5 \mathrm{M})$ at $190^{\circ} \mathrm{C}$ (entry 1$)$, afforded 2 in only $6 \%$ yield. Following this result, the effect of varying reaction temperatures was investigated $\left(70-170{ }^{\circ} \mathrm{C}\right.$, entry 2 and Supplementary Information). While temperatures significantly above $170{ }^{\circ} \mathrm{C}$ or below $70{ }^{\circ} \mathrm{C}$ only led to trace amounts of $\mathbf{2}$, maintaining the reaction temperature at $100^{\circ} \mathrm{C}$ improved the yield of 2 to $37 \%$. The presence of zinc chloride or acetic acid (entries 3 and 4), which are common additives in the related Fischer-indole synthesis, was not beneficial.

Table 1: Conversion of $\mathbf{1}$ to $\mathbf{2}$ under varying reaction conditions.

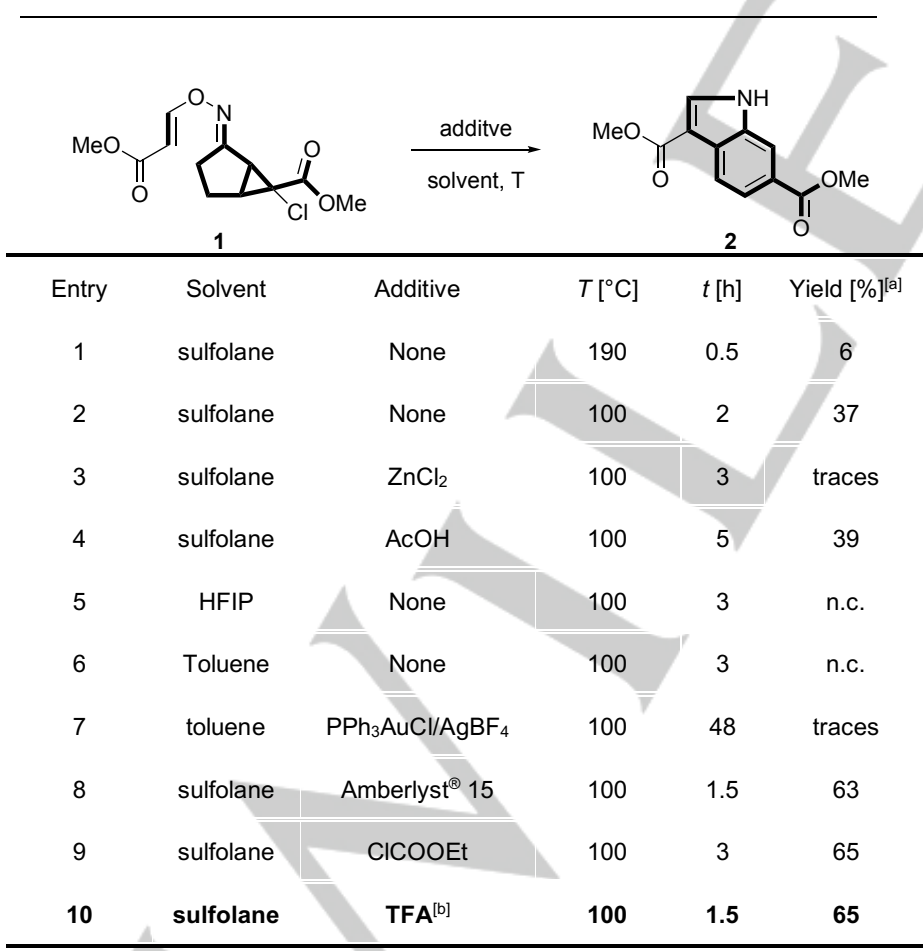

[a] Yield of the isolated product. [b] 1.0 equiv TFA. TFA = trifluoroacetic acid, $\mathrm{HFIP}=1,1,1,3,3,3$-hexafluoro-2-propanol.
A survey of solvents revealed that hexafluoroisopropanol (HFIP) and toluene were ineffective, with only starting material being recovered (entries 5 and 6). In 2010, the Camp group reported a gold(I) catalyzed rearrangement of $O$-vinyl oximes to give substituted pyrroles. ${ }^{[11]}$ Unexpectedly, when 1 was subjected to these conditions, indole $\mathbf{2}$ was only produced in trace amounts (entry 7). However, significantly improved yields were achieved by employing Amberlyst ${ }^{\circledR} 15$ as a proton source (63\% yield, entry 8$)$. In an attempt to combine the indole formation with an in situ $\mathrm{N}$-protection, we heated a mixture of 1 and ethyl chloroformate in dry sulfolane at $100{ }^{\circ} \mathrm{C}$ (entry 9). Surprisingly, only formation of unprotected 2 (65\%) was observed in this case. An identical yield was obtained when ethyl chloroformate was replaced with trifluoroacetic acid (entry 10). Although the exact role of the acid (or chloroformate) additive is unclear at this point, we believe that it simply increases the rate of imine to enamine tautomerization and thus facilitates initiation of the cascade reaction.

With the optimized conditions in hand, we applied our methodology to a variety of O-vinyl oximes, which were prepared in three steps from readily available cyclopenten-2ones (Figure 3 ). The reactions could be conducted in an openflask, with generally short reaction times $(<3 \mathrm{~h})$, and the presence of one equivalent of trifluoracetic acid was found to be optimal in all cases.
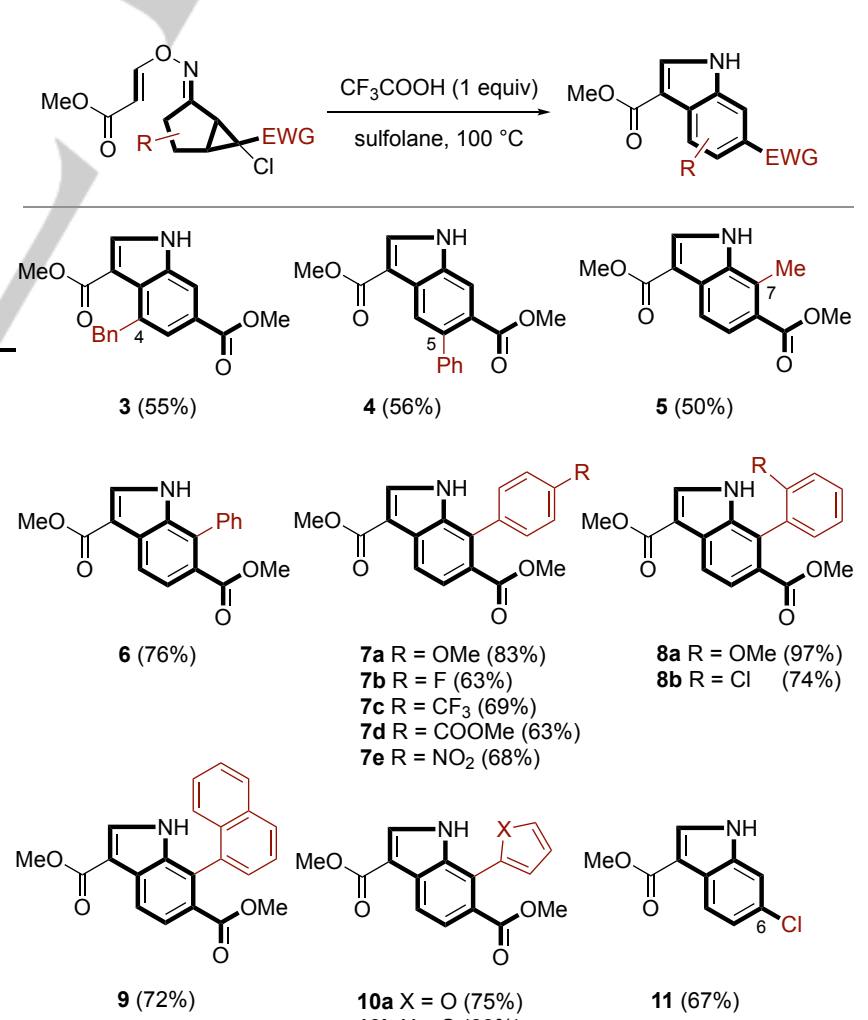

Figure 3. Synthesis of indoles and variation of the substitution pattern at C4C7. 
As demonstrated for $\mathbf{3}$ and $\mathbf{4}$, substituents at the C4 and C5 position of the indole are well tolerated. We could further show that a variety of C7-substituted indoles (5-10) are accessible and that the transformation is not limited to aryl substituents (5). While indoles bearing a para-substituted C7aryl group (compounds $\mathbf{7 a - 7 e )}$ were produced in yields up to $83 \%$, indoles bearing an ortho-substituted C7-aryl group (8a and 8b) were isolated in excellent yields up to $97 \%$. The method also worked well for naphthyl-substituted indole $\mathbf{9}$ and with heteroaromatic substituents such as 2-furyl (10a) and 2-thienyl (10b). To further vary the substitution pattern of the C6-position of the indole core, we also investigated the gem-dichloro cyclopropane derivative of $O$-vinyl oxime 1 . With this substrate, we obtained the 6 -chloro indole 11 in $67 \%$ yield.

Mechanistically, we envisioned that the enamine tautomer $1^{\text {[11] }}$ would follow one of the two pathways illustrated in Scheme 1 to give indole 2. In pathway A, a [3,3]-rearrangement produces the pyrrol intermediate 12 , which is then converted to 2 via a thermally induced $2 \pi$-disrotatory ring-opening-aromatization sequence. In pathway $B$, the strain-releasing, ring-openingaromatization reaction takes place first to give the aryl O-vinyl oxime 13 which undergoes a sequential [3,3]-rearrangement and condensation to deliver indole 2.

Based on literature precedent, ${ }^{[11,12 a]}$ it seemed reasonable that that either of the two pathways could be operational, but it was unclear whether any of the proposed intermediates could be detected by NMR spectroscopy. Interestingly, when a solution of 1 and trifluoroacetic acid (1 equiv) in sulfolane was monitored by ${ }^{1} \mathrm{H}$ NMR spectroscopy and was gradually heated from $30^{\circ} \mathrm{C}$ to $100^{\circ} \mathrm{C}$, we observed neither intermediate 12 nor intermediate 13. After $1 \mathrm{~h}$, heating was discontinued and indole 2 could be isolated as the sole product in $65 \%$ yield.

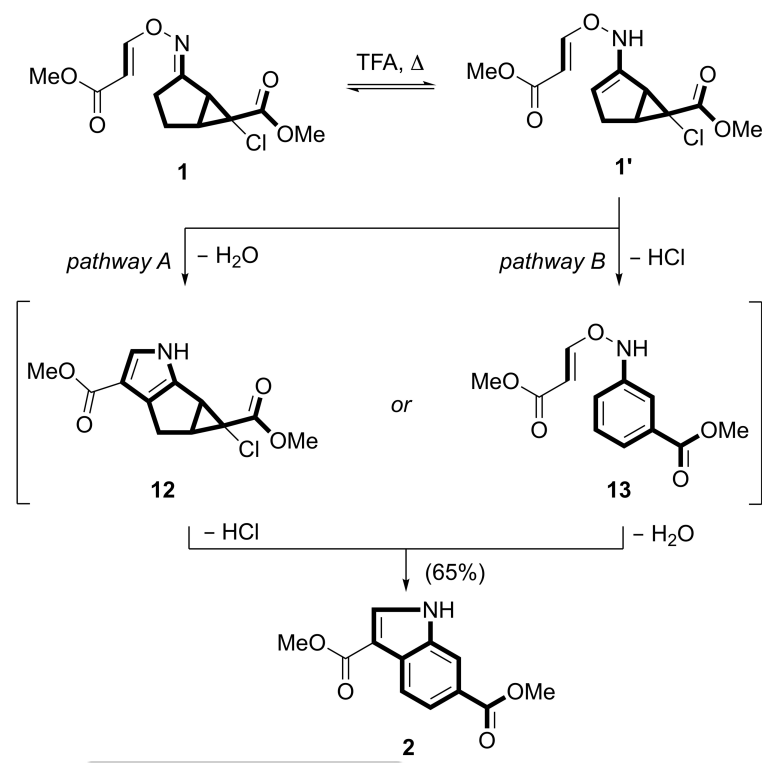

Scheme 1. Mechanistic hypotheses for the indole formation.
Since monitoring of the reaction by ${ }^{1} \mathrm{H}$ NMR spectroscopy did not allow us to differentiate between the two pathways, we next focused our attention on the synthesis of the modified components 14 and 17 (Scheme 2). First, we prepared unsubstituted cyclopropane fragment 14, lacking the crucial chloride-leaving group for the ring-expansion reaction. Surprisingly, exposure of this substrate to trifluoroacetic acid in sulfolane at $30^{\circ} \mathrm{C}$ for $14 \mathrm{~h}$ did not afford pyrrole 15 , but left the starting material unreacted. At elevated temperatures $\left(100{ }^{\circ} \mathrm{C}\right)$, complete decomposition of the substrate occurred within $3 \mathrm{~h}$.

Next, we attempted to synthesize the aryl O-vinyl oxime 17 via 1,4-diazabicyclo[2.2.2]octane (DABCO) catalyzed 1,4addition of $\mathrm{N}$-aryl hydroxylamine $\mathbf{1 6}$ to methyl propiolate at $-10^{\circ} \mathrm{C}$. However, using DABCO in the 1,4 -addition step, did not afforded 17 but rather directly gave rise to the corresponding indole 18. ${ }^{[12 b]}$ Based on these results, we concluded that the mechanism of the indole formation most likely proceeds via pathway B.
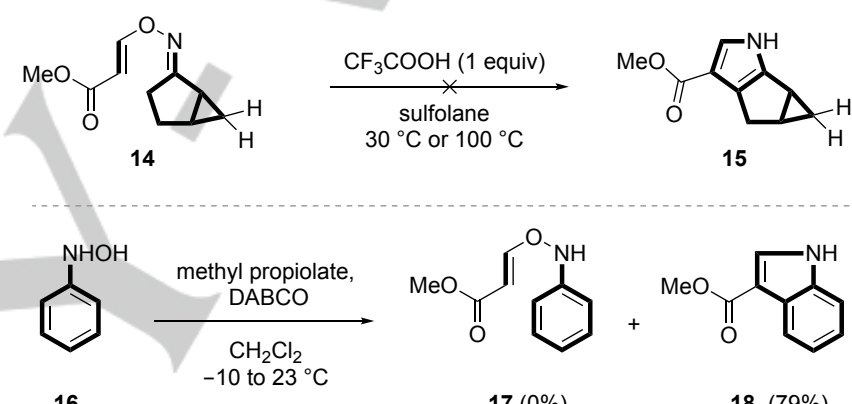

16

$17(0 \%)$

$18(79 \%)$

Scheme 2. Mechanistic probes for the indole formation. $\mathrm{DABCO}=1,4-$ diazabicyclo[2.2.2]octane.

Having established a versatile platform for the synthesis of indoles, we turned our attention to the preparation of substituted indazoles. After intensive screening of reaction conditions, we were pleased to find that the condensation reaction of diketone 19 with hydrazine monohydrochloride in a sealed tube directly afforded 20. Simple variation of diketone 19 gave access to a variety of C3-substituted indazoles, which are depicted in Figure 4. Indazoles bearing an aliphatic (21 and 22), phenyl (23) or methoxymethyl (24) group were obtained in yields up to $77 \%$. Electron-deficient substituents, such as methyl ester (25a and 25b) or trifluoromethyl (26) groups, and heteroaromatic substituents, such as 2-pyridinyl (27), 2-furyl (28) or 2-thienyl (29) were also well tolerated. In sharp contrast to the indole synthesis, formation of the related indazoles could also be conducted in a stepwise manner to first form a pyrazole at 23 ${ }^{\circ} \mathrm{C}$. Upon heating to $100{ }^{\circ} \mathrm{C}$, full conversion to the indazole was observed.

Having developed an efficient strategy for the synthesis of C6-substituted indazoles, we set out to further extend our de novo platform for the construction of other benzannelated fivemembered heterocycles. Exposure of 1,4-diketone $\mathbf{3 0}$ and $\mathbf{3 2}{ }^{[13]}$ to trifluoroacetic acid at $190{ }^{\circ} \mathrm{C}$ initiated the ring- 
expansion/aromatization cascade reaction to provide in good yields benzofurans 31 and 33 , respectively. Alternatively, the use of Lawesson's reagent $\mathbf{3 6}$ in the cyclization reaction gave access to benzothiophenes 34 and 35 , respectively.

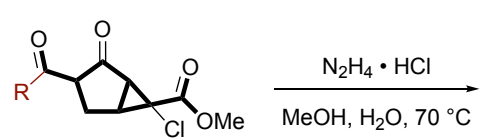

19<smiles>CCc1n[nH]c2cc(C(=O)OC)ccc12</smiles>

$21(72 \%)$<smiles>COCc1n[nH]c2cc(C(=O)OC)ccc12</smiles>

$24(59 \%)$<smiles>COC(=O)c1ccc2c(-c3ccccn3)n[nH]c2c1</smiles>

$27(65 \%)$ 25a EWG $=$ COOMe $(53 \%)$ 25b EWG $=\mathrm{Cl}(52 \%)^{[a]}$
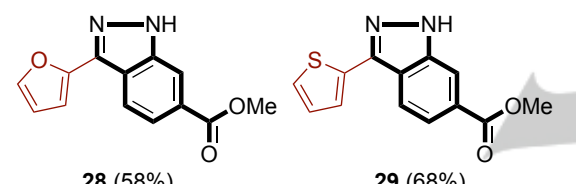

$29(68 \%)$<smiles>COC(=O)c1ccc2c(C(F)(F)F)n[nH]c2c1</smiles>

$26(65 \%)$

Figure 4. One-Pot Synthesis of substituted indazoles from bicyclo[3.1.0]hexan-2-ones. [a] Yield over two steps after chromatographic purification.

\section{Conclusions}

In conclusion, we have developed a protocol for the de novo construction of polysubstituted indoles, indazoles, benzofurans and benzothiophens from non-aromatic precursors. The reaction can be conducted in an open-flask, is operationally simple and does not require expensive reagents or catalysts. Many of the presented heterocycles feature a unique substitution pattern and have never been synthesized before. A broad application of the developed strategy for the synthesis of highly substituted benzannelated heterocycles and its implementation in complex molecule syntheses are currently underway in our laboratory.

\section{Experimental Section}

Experimental details and compound characterization data and copies of ${ }^{1} \mathrm{H}$ and ${ }^{13} \mathrm{C}$ NMR spectra, are available in the Supporting Information.

\section{Acknowledgements}

We gratefully acknowledge financial support from the German Research Foundation (Emmy Noether Project MA5999/2-1), the European Research Council (ERC StG 714049) and the Funds of the Chemical Industry (Sachkostenzuschuss and Dozentenpreis). We thank Dr. Cedric Hugelshofer (UC Berkeley) and Dr. Kevin Mellem (Revolution Medicines) for helpful discussions during the preparation of this manuscript.

Keywords indoles $\bullet$ indazoles $\cdot$ benzofuran $\bullet$ cyclopropanes $•$ ring-expansion

[1] a) B. Schäfer, Naturstoffe in der chemischen Industrie, Elsevier, München, 2007; b) for total syntheses of verruculogen see: Y. Feng, D. Holte, J. Zoller, S. Umemiya, L. R. Simke, P. S. Baran, J. Am. Chem. Soc. 2015, 137, 10160-10163; c) W. Zhang, C. Yang, C. Huang, L. Zhang, H. Zhang, Q. Zhang, C. Yuan, Y. Zhu, C. Zhang, Org. Lett, 2017, 19, 592-595; d) for a total synthesis of mitomycin K see: J. W. Benbow, G. K. Schulte, S. J. Danishefsky, Angew. Chem. Int. Ed. 1992, 31, 915-917.

[2] J. A Joule, K. Mills, Heterocyclic chemistry 5th ed., John Wiley \& Sons, Chichester 2010.

[3] K. Hayakawa, T. Yasukouchi, K. Kanematsu, Tetrahedron Lett. 1986, 27, 1837-1840.

[4] C. L. Morales, B. L. Pagenkopf, Org. Lett., 2008, 10, 157-159.

[5] T. J. Greshock, R. L. Funk, Org. Lett., 2006, 8, 2643-2645.

[6] For syntheses of indazoles from cyclohexanones see: a) C. Ainsworth, Org. Synth. 1959. 39, 27; b) S. Tsukamoto, S. Sakamoto, F. Wanibuchi, Y. Naitou, K. Hatanaka, Y. Kimura, T. Kimizuka, H. Kubota, K. Kazuta, K. Maeno, I. Shimada, Bioorg. Med. Chem. 2008, 16, 1966-1982.

[7] For selected reviews see: a) P. K. Tirunahari, D. F. Taber, Tetrahedron, 2011, 67, 7195-7210; b) G. R. Humphrey, J. T. Kuethe, Chem. Rev. 2006, 106, 2875-2911. 
[8] J. Feierfeil, A. S. Grossmann, T. Magauer, Angew. Chem. Int. Ed. 2015, 54, 11835-11838.

[9] T. A. Unzner, A. S. Grossmann, T. Magauer, Angew. Chem. Int. Ed. 2016, 55, 9763-9767.

[10] O-Vinyl oxime 1 was obtained as an inconsequential mixture of double bond isomers. The depicted isomer is the major double bond isomer.

[11] Pyrrol synthesis via gold catalyzed rearrangement of $O$-vinyl oximes: S. Ngwerume, J. E. Camp, Chem. Commun. 2011, 47, 1857-1859.

[12] a) M. O. Gray, R. J. Lin, H. V. Patel, J. R. Hwu, J. Org. Chem, 1994, 59, $1577-1582 ;$ b) In our hands, conducting the 1,4-addition of $\mathrm{N}$-aryl hydroxylamine to methyl propiolate using DMAP did not lead to the corresponding indole. These results are contrary to those reported in the literature, see reference 12a. The use of methyl 3(hydroxyamino)benzoate led to a mixture of regioisomeric indoles, which could not be fully characterized.

[13] For the synthesis of 1,4-diketones see: R. Ruzziconi, A. Casu, E. Baciocchi, Tetrahedron Lett. 1989, 30, 3707-3710. 


\section{FULL PAPER}

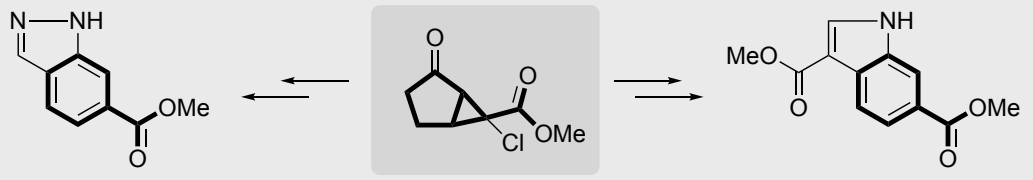

Here we describe a powerful cascade transformation for de novo construction of variously substituted indoles, indazoles, benzofurans and benzothiophenes. The transformation can be conducted under mild, non-anhydrous conditions. For the synthesis of indoles, mechanistic studies revealed that the electrocyclic ringopening of the bicyclic ring-system and aromatization precedes the 3,3-sigmatropic rearrangement.
Johannes Feierfeil, Thomas Magauer*

Page No. - Page No.

De Novo Synthesis of Benzannelated Heterocycles 